\title{
Effect of Efflux Pump Inhibitor Carbonyl Cyanide3-Chlorophenylhydrazone on the Minimum Inhibitory Concentration of Ciprofloxacin in Acinetobacter baumannii Clinical Isolates
}

\author{
Abdollah Ardebili ${ }^{1,2}$; Malihe Talebi ${ }^{1,2}$; Leila Azimi ${ }^{2}$; Abdolaziz Rastegar Lari ${ }^{1,2,}$ \\ ${ }^{1}$ Department of Microbiology, Faculty of Medicine, Iran University of Medical Sciences, Tehran, IR Iran \\ ${ }^{2}$ Antimicrobial Resistance Research Center, Iran University of Medical Sciences, Tehran, IR Iran \\ ${ }^{*}$ Corresponding author: Abdolaziz Rastegar Lari, Department of Microbiology, School of Medicine, Iran University of Medical Sciences, Tehran, IR Iran. Tel: +98-2186703181, Fax: +98- \\ 2186703183, E-mail:azizlari@gmail.com
}

Received: October 21, 2012; Revised: December 18, 2012; Accepted: January 27, 2013

\begin{abstract}
Background: Acinetobacter baumannii is an important human pathogen with increasing notoriety in the recent years, as a causative organism of drug resistant nosocomial infections, particularly in immunocompromised patients hospitalized in burn centers.

Objectives: The aim of this study was to determinate the role of efflux pump(s) in ciprofloxacin resistance of A. baumannii strains isolated from burn patients.

Materials and Methods: Sixty-five A. baumannii strains were isolated from the burn patients hospitalized in Motahari Burns and Reconstruction Center in Tehran, Iran. Susceptibility test to ciprofloxacin was carried out by disk agar diffusion and agar dilution methods, according to the CLSI guidelines. Activity of the efflux system was evaluated using efflux pump inhibitor carbonyl cyanide 3-chlorophenylhydrazone (CCCP).

Results: All Acinetobacter isolates were resistant to ciprofloxacin. The Minimum inhibitory concentration (MIC) range of ciprofloxacin in isolates was 4 to $128 \mu \mathrm{g} / \mathrm{mL}$ or greater. Moreover, susceptibility of strains to ciprofloxacin was highly increased in the presence of efflux pump inhibitor; So that, for $86.1 \%$ (56/65) of isolates, CCCP reduced the MIC by 2 to 64 folds.

Conclusions: Our findings are suggestive that efflux-based system may play a role in fluoroquinolone resistance in A. baumannii isolates, affecting hospitalized patients. The ability of Acinetobacter to acquire resistance to these potent antimicrobials by the efflux pump mechanism is a concern. Therefore, new strategies are required in order to eliminate the efflux transport activity from the resistant bacteria causing nosocomial infections and provide more appropriate approaches for treatment and management of troubling infections.
\end{abstract}

Keywords: Acinetobacter baumannii; Burn; Ciprofloxacin; Efflux Pumps; Carbonyl cyanide 3-chlorophenylhydrazone

\section{Background}

Over the past three decades, Acinetobacter baumannii has emerged as a serious nosocomial pathogen, especially in hospitalized burned patients worldwide (1). Today, this bacterium and Pseudomonas aeruginosa are the predominant isolated organisms of burn patients in many countries, including Iran (2). Certain strains of A. baumannii are now resistant to many commonly-used antibiotics, including fluoroquinolones, and multidrug resistance is often responsible for the failure of antibiotic therapy (3, 4). Resistance to fluoroquinolones is mediated mainly by chromosomal mutations in gyrA and parC genes that are associated with high levels of resistance $(5,6)$. Another mechanism responsible for fluoroquinolones resistance is reduction in the drug accumulation due to either impermeability of outer membrane or overexpression of active efflux pumps (1, 7-10).

Efflux systems are composed of transport proteins that pump out a broad range of toxic substrates such as antibiotics and biocides from bacteria, in an energy-dependent manner (6). In these circumstances, the intracellular antibiotic concentration is decreased and bacteria become less susceptible to that compound. The minimum inhibitory concentrations (MICs) of antibiotics for strains overexpressing an efflux pump, are usually 2 to 8 folds higher than those for susceptible strains of that species $(10,11)$.

To assess the role of drug efflux mechanism in bacteria, efflux pump inhibitors (EPIs) are widely used to totally abolish the efflux of various molecules $(10,12)$. One of these compounds is carbonyl cyanide 3-chlorophenylhydrazone (CCCP) (an uncoupler of oxidative phosphorylation which disrupts the proton

Implication for health policy/practice/research/medical education:

This manuscript can inform infectious disease specialists about the antibiotic resistance status in problematic bacteria, including A. baumannii, and help choose a newer proper antibiotic(s) for treatment of related bacterial infections.

Copyright (C) 2014,Ahvaz Jundishapur University of Medical Sciences; Published by Kowsar Corp. This is an open-access article distributed under the terms of the Creative Commons Attribution License, which permits unrestricted use, distribution, and reproduction in any medium, provided the original work is properly cited. 
gradient of the membranes) that has often been found to increase the susceptibility of a number of multidrug resistant bacteria, including A. baumannii $(9,13,14)$.

\section{Objectives}

There are several documented studies on fluoroquinolones resistance in clinical isolates of $A$. baumannii from Iranian populations, but the role of A. baumannii efflux pump in resistance to fluoroquinolones has not been investigated. So, the aim of this study was to analyze the contribution of active efflux system to ciprofloxacin resistance in clinical isolates of $A$. baumannii using the efflux pump inhibitor CCCP.

\section{Materials and Methods}

\subsection{Patients and Bacterial Isolates}

In a six-month study, a total of 164 burn patients, hospitalized at least 2 weeks at Motahari Burns and Reconstruction Center in Tehran, were participated. The age range of the patients was between 1 and 88 years, the burn degree was at least II, and in most of them, the total body surface area (TBSA) was more than $10 \%$. After sampling the burn wounds, clinical specimens were examined microbiologically. Bacterial isolates were identified as A. baumannii based on the standard biochemical tests following the criteria of Bouvet and Grimont (15) and then confirmed by PCR amplification of blaOXA-51-like gene (Figure 1) (16 $-18)$.

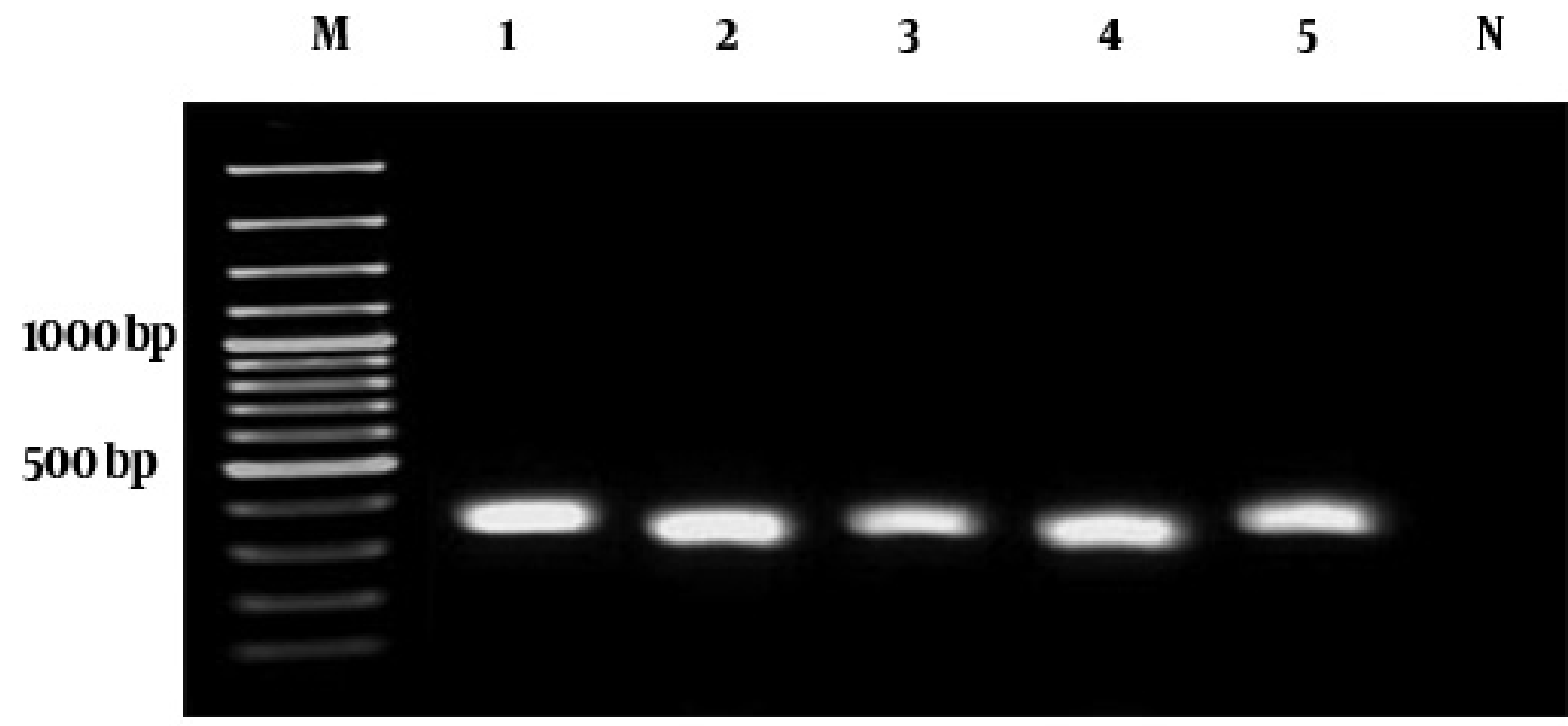

Lanes: M, 100 bp DNA Ladder; 1-5, PCR products of blaOXA-51-like gene (353 bp); N, negative control (A. lwoffii).

\subsection{PCR Amplification of blaOXA-51-Like Gene}

PCR analysis of blaOXA-51-like carbapenemase gene was used in order to confirm the presence of $A$. baumannii species (16). Extraction of genomic DNA from bacterial isolates was done by the genomic DNA purification kit (Fermentas, Germany) according to the manufacturer's instructions. The primer pair, 5'-TAATGCTTTGATCG GCCTTG-3' and 5'-TGGATTGCACTTCATCTTGG-3' was used for gene amplification. The PCR conditions were as follows: initial denaturation at $94^{\circ} \mathrm{C}$ for 5 minutes; 30 cycles with denaturation at $94^{\circ} \mathrm{C}$ for 45 seconds, annealing at $58^{\circ} \mathrm{C}$ for 1 minute, and extension at $72^{\circ} \mathrm{C}$ for 1 minute, followed by final extension at $72^{\circ} \mathrm{C}$ for 5 minutes. The PCR products were detected by electrophoresis in agarose gel $(2, \mathrm{w} / \mathrm{v} \%)$ containing $0.5 \mu \mathrm{g} / \mathrm{mL}$ of ethidium bromide.

\subsection{Antimicrobial Susceptibility Testing}

Susceptibility of the A. baumannii isolates to ciprofloxacin $(5 \mu \mathrm{g})$ (Mast, Merseyside, UK) was initially tested using the standard disk agar diffusion method on MuellerHinton (M-H) agar plates. Then, the MIC of ciprofloxacin 
against bacterial isolates was evaluated by the agar dilution technique. Both of these methods were carried out according to the guidelines established by the clinical and laboratory standards institute (CLSI) (19). The standard strain of A. baumannii 19606 was used for quality control of the susceptibility studies.

\subsection{Treatment of the Efflux Pump Inhibitor}

To explore the presence of efflux pump mechanism, efflux pump inhibitor CCCP was added to each of $\mathrm{M}-\mathrm{H}$ agar plates containing 0.5 to $128 \mu \mathrm{g} / \mathrm{mL}$ ciprofloxacin. The final concentration of CCCP in the M-H agar was $25 \mu \mathrm{g} / \mathrm{mL}$ (20). Then, MIC for ciprofloxacin was determined again. A plate containing CCCP and not containing antibiotics was used as control. The positive criterion for the presence of efflux pump in isolates was decrease of at least 4 folds of ciprofloxacin MIC after the CCCP addition (20).

\section{Results}

\subsection{Bacterial Isolates and Ciprofloxacin Resistance}

In a six months period, 65 strains of A. baumannii were isolated from hospitalized burn patients using biochemical tests and confirmatory PCR assays. The PCR-amplified DNA products of blaOXA-51-like carbapenemase gene from five selected clinical isolates are shown in Figure 1. The preliminary results of ciprofloxacin susceptibility test using the disk agar diffusion method showed that all of the A. baumannii strains were resistant to this antibiotic. The MIC for ciprofloxacin in bacterial isolates is shown in Table 1. According to the established breakpoint values recommended by CLSI (19), the A. baumannii isolates with MIC $\geq 4 \mu \mathrm{g} / \mathrm{mL}$ are considered as ciprofloxacin resistant. In the present study, Acinetobacter isolates had a ciprofloxacin MIC range between 4 to $128 \mu \mathrm{g} / \mathrm{mL}$ or greater; so that, the MICs for 6.1\% (4/65), 43\% (28/65) and 10.7\% (7/65) of isolates were 4,128 and $>128 \mu \mathrm{g} / \mathrm{mL}$, respectively.

Table 1. Effects of CCCP on the Ciprofloxacin MIC in A. baumannii Isolates

\begin{tabular}{|c|c|c|c|}
\hline $\begin{array}{l}\text { Isolates, } \\
\text { No. (\%) }\end{array}$ & $\begin{array}{l}\text { MIC }^{a}-{ }_{-C C P}{ }^{a} \\
\mu \mathrm{g} / \mathbf{m L}\end{array}$ & $\begin{array}{l}\text { MIC Ranges, } \\
\mu \mathrm{g} / \mathrm{mL}+\mathrm{CCCP}\end{array}$ & $\begin{array}{l}\text { Fold Reduction } \\
\text { in } M I C+C C C P\end{array}$ \\
\hline $4(6.1)$ & 4 & $1-2$ & $2-4$ \\
\hline $3(4.6)$ & 8 & $1-4$ & $2-8$ \\
\hline $1(1.5)$ & 16 & 8 & 2 \\
\hline $7(\mathbf{1 0 . 7 )}$ & 32 & $4-32$ & $0-8$ \\
\hline $15(23)$ & 64 & $1-64$ & $0-64$ \\
\hline $28(43)$ & 128 & $32-128$ & $0-4$ \\
\hline $7(\mathbf{1 0 . 7})$ & $>128$ & $32-128$ & At least 2 \\
\hline
\end{tabular}

\subsection{Effects of the Efflux Pump Inhibitor on} Ciprofloxacin Resistance

To determinate the role of efflux pump in the ciprofloxacin resistant phenotypes in 65 A. baumannii isolates, we evaluated the MIC of ciprofloxacin in the presence of $25 \mu \mathrm{g} / \mathrm{mL}$ CCCP, and then, compared the MICs with and without CCCP. Results indicated that most of the isolates (86.1\%) became less resistant ( 2 to 64 folds) to ciprofloxacin in the presence of efflux pump inhibitor (Table 1). Based on a 4 -fold or greater decrease in the MIC as the criterion for significance (15), the MIC for 30 of the 65 isolates (46.1\%) was reduced significantly 4 to 64 folds. In addition, when the efflux pump inhibitor was added, 26 isolates (40\%) exhibited a 2-fold MIC change, while 9 (13.8\%) did not change. All bacteria grew well in the $\mathrm{M}-\mathrm{H}$ agar plates with CCCP that did not contain ciprofloxacin, indicating that $25 \mu \mathrm{g} / \mathrm{mL}$ CCCP did not have an antibacterial effect itself. These results showed that the drug efflux systems are associated with resistance to ciprofloxacin in A. baumannii isolates.

\section{Discussion}

Acinetobacter species, especially A. baumannii, are responsible for hospital-acquired infections. Nowadays, antimicrobial resistance in this bacterium has become an important issue worldwide. Resistance rates to most antibiotics such as fluoroquinolones, as one of the firstline drugs to treat $A$. baumannii infections, are increasing globally, including in Iran (21-24). In a survey conducted by Wang and colleagues in Taiwan, all A. baumannii isolates were resistant to ciprofloxacin and other antibiotics (21). Additionally, in a six-year prospective study in Iran, it was found that only $20.1 \%$ of $A$. baumannii isolates were susceptible to ciprofloxacin and susceptibility rate to this antibiotic reduced gradually among Acinetobacter isolates in Iran (22).

Asadollahi et al. during 2009 and 2010 determined that $100 \%$ of $A$. baumannii isolates were resistant to ciprofloxacin (25). Similar to the mentioned studies, all of our isolates showed resistance to ciprofloxacin. However, resistance rate observed in the present investigation was lower than that of UK and China (50.9\% and $61.2 \%$, respectively) $(4,26)$. This discrepancy could be due to differences in the quality programs of antimicrobial susceptibility, patterns of antibiotic usage, geographic conditions, and environmental factors in various countries. Furthermore, given the MIC values in our study, similar to those reported by Valentine et al. (3), emergence of high-level resistant A. baumannii strains to ciprofloxacin among the hospitalized burn patients in Tehran is of concerns.

There are increasing evidences that drug efflux pumps are a mechanism of resistance in a number of clinically-important bacteria, including A. baumannii (7-11). Although high level resistance may not occur as a result 
of multidrug efflux pumps alone, the overexpression association of these genes among highly-resistant clinical isolates cannot be ignored. Efflux pump inhibitors have been shown to reverse multidrug resistance in $A$. baumannii and other bacteria (10). The effects of these compounds, such as CCCP, on the antimicrobial susceptibility were examined in some studies $(1,27)$. Rajamohan and coworkers found that addition of CCCP at final concentration of $25 \mu \mathrm{g} / \mathrm{mL}$ greatly reduced the MIC of various biocides from 2 to 12 folds (27). In consistence with the results obtained by Lin et al. (9), we observed that the ciprofloxacin susceptibility of most isolates was increased in the presence of CCCP, mainly 2 to 4 folds.

These results suggest that drug efflux pumps are involve in resistance to fluoroquinolone in clinical isolates of A. baumannii. AdeABC is an efflux pump described in A. baumannii, overexpression of which confers resistance to fluoroquinolones and other antimicrobial agents. Further studies are necessary to clarify the role of this efflux pump overexpression on fluoroquinolone resistance in $A$. baumannii (8-10). In this regard, detection of the AdeABC efflux pump by PCR and determination of the role of its alterations in fluoroquinolone resistance of Acinetobacter strains will be a part of our researches in the future.

In conclusion, it seems from the present and other local studies that the prevalence of fluoroquinolone-resistant A. baumannii strains has recently increased in Iranian hospitals. Furthermore, results of our investigation are suggestive that drug efflux system has a role in conferring resistance to fluoroquinolone in Acinetobacter isolates and this mechanism is getting widespread in clinical settings as well as among hospitalized patients, especially in burn units. So, efforts should be aimed at detecting such resistant bacteria along with their resistance mechanisms, controlling infections caused by them, and finally, providing better alternative therapies against these recalcitrant organisms.

\section{Acknowledgements}

Authors would like to thank the staff of Antimicrobial Resistance Research Center for supporting this study.

\section{Authors' Contribution}

Dr. Abdolaziz Rastegar Lari supervised the project and contributed to the analysis of data. Dr. Malihe Talebi analyzed and abstracted the data. Abdollh Ardebili developed the original idea and the protocol, analyzed and interpreted the data and wrote the manuscript. Leila Azimi contributed to the development of the protocol.

\section{Financial Disclosure}

All authors declare that they have no conflict of interest.

\section{Funding/Support}

This study was funded by Iran University of Medical Sciences (Grant no.1067).

\section{References}

1. Park S, Lee KM, Yoo YS, Yoo JS, Yoo JI, Kim HS, et al. Alterations of gyrA, gyrB, and parC and Activity of Efflux Pump in Fluoroquinolone-resistant Acinetobacter baumannii. Osong Public Health Res Perspect. 2011;2(3):164-70.

2. Alaghehbandan R, Azimi L, Rastegar Lari A. Nosocomial infections among burn patients in Teheran, Iran: a decade later. Ann Burns Fire Disasters. 2012;25(1):3-7.

3. Valentine SC, Contreras D, Tan S, Real LJ, Chu S, Xu HH. Phenotypic and molecular characterization of Acinetobacter baumannii clinical isolates from nosocomial outbreaks in Los Angeles County, California. J Clin Microbiol. 2008;46(8):2499-507.

4. Spence RP, Towner KJ. Frequencies and mechanisms of resistance to moxifloxacin in nosocomial isolates of Acinetobacter baumannii. J Antimicrob Chemother. 2003;52(4):687-90.

5. Vila J, Ruiz J, Goni P, Jimenez de Anta T. Quinolone-resistance mutations in the topoisomerase IV parC gene of Acinetobacter baumannii. J Antimicrob Chemother. 1997;39(6):757-62.

6. Hamouda A, Amyes SG. Novel gyrA and parC point mutations in two strains of Acinetobacter baumannii resistant to ciprofloxacin. J Antimicrob Chemother. 2004;54(3):695-6.

7. Kumar A, Schweizer HP. Bacterial resistance to antibiotics: active efflux and reduced uptake. Adv Drug Deliv Rev. 2005;57(10):1486513.

8. Higgins PG, Wisplinghoff H, Stefanik D, Seifert H. Selection of topoisomerase mutations and overexpression of adeB mRNA transcripts during an outbreak of Acinetobacter baumannii. $J$ Antimicrob Chemother. 2004;54(4):821-3.

9. Lin L, Ling BD, Li XZ. Distribution of the multidrug efflux pump genes, adeABC, adeDE and adeIJK, and class 1 integron genes in multiple-antimicrobial-resistant clinical isolates of Acinetobacter baumannii-Acinetobacter calcoaceticus complex. Int Antimicrob Agents. 2009;33(1):27-32.

10. Zechini B, Versace I. Inhibitors of multidrug resistant efflux systems in bacteria. Recent Pat Antiinfect Drug Discov. 2009;4(1):37-50

11. Piddock LJ. Clinically relevant chromosomally encoded multidrug resistance efflux pumps in bacteria. Clin Microbiol Rev. 2006;19(2):382-402.

12. Mahamoud A, Chevalier J, Alibert-Franco S, Kern WV, Pages JM Antibiotic efflux pumps in Gram-negative bacteria: the inhibitor response strategy. J Antimicrob Chemother. 2007;59(6):1223-9.

13. Nikasa P, Abdi-Ali A, Rahmani-Badi A, Al-Hamad A. In vitro Evaluation of Proton Motive Force-Dependent Efflux Pumps Among Multidrug Resistant Acinetobacter baumannii Isolated From Patients at Tehran Hospitals. Jundishapur J Microbiol. 2013;6(7): e6792.

14. Huang L, Sun L, Xu G, Xia T. Differential susceptibility to carbapenems due to the AdeABC efflux pump among nosocomial outbreak isolates of Acinetobacter baumannii in a Chinese hospital. Diagn Microbiol Infect Dis. 2008;62(3):326-32.

15. Bouvet PJM, Grimont PAD. Taxonomy of the Genus Acinetobacter with the Recognition of Acinetobacter baumannii sp. nov., Acinetobacter haemolyticus sp. nov., Acinetobacter johnsonii sp. nov., and Acinetobacter junii sp. nov. and Emended Descriptions of Acinetobacter calcoaceticus and Acinetobacter lwoffii. Int $J$ System Bacteriol.1986;36(2):228-240.

16. Turton JF, Woodford N, Glover J, Yarde S, Kaufmann ME, Pitt TL. Identification of Acinetobacter baumannii by detection of the blaOXA-51-like carbapenemase gene intrinsic to this species. $J$ Clin Microbiol. 2006;44(8):2974-6.

17. Farahani RK, Moniri R, Dastehgoli K. Multi-Drug Resistant Acinetobacter-Derived Cephalosporinase and OXAs-etC Genes in 
Clinical Specimens of Acinetobacter spp. Isolated From Teaching Hospital. Jundishapur J Microbiol. 2013;6(2):181-185.

18. Opazo A, Dominguez M, Bello H, Amyes SG, Gonzalez-Rocha G. OXA-type carbapenemases in Acinetobacter baumannii in South America. J Infect Dev Ctries. 2012;6(4):311-6.

19. Clinical and Laboratory Standards Institute. Performance standards for antimicrobial susceptibility testing; Approved standard.PA, USA: Wayne; 2010.

20. Pumbwe L, Glass D, Wexler HM. Efflux pump overexpression in multiple-antibiotic-resistant mutants of Bacteroides fragilis. Antimicrob Agents Chemother. 2006;50(9):3150-3.

21. Wang SH, Sheng WH, Chang YY, Wang LH, Lin HC, Chen ML, et al. Healthcare-associated outbreak due to pan-drug resistant Acinetobacter baumannii in a surgical intensive care unit. J Hosp Infect. 2003;53(2):97-102.

22. Soroush S, Haghi-Ashtiani MT, Taheri-Kalani M, Emaneini M, Aligholi M, Sadeghifard N, et al. Antimicrobial resistance of nosocomial strain of Acinetobacter baumannii in Children's Medical Center of Tehran: a 6-year prospective study. Acta Med Iran. 2010;48(3):178-84.

23. Mostofi S, Mirnejad R, Masjedian F. Multi-drug resistance in
Acinetobacter baumannii strains isolated from clinical specimens from three hospitals in Tehran-Iran. Afr J Microbiol Res. 2011;5(21):3579-3582.

24. Mansouri S, Razavi M, Norouzi F, Najar SG. Prevalence of $\beta$-Lactamase Production and Antimicrobial Susceptibility of Multidrug Resistant Clinical Isolates of Non-Fermenting Gram Negative Bacteria From Hospitalized Patients in Kerman/Iran. Jundishapur J Microbiol. 2012;5(2):405-410.

25. Asadollahi P, Akbari M, Soroush S, Taherikalani M, Asadollahi K, Sayehmiri K, et al. Antimicrobial resistance patterns and their encoding genes among Acinetobacter baumannii strains isolated from burned patients. Burns. 2012;38(8):1198-1203.

26. Wei-feng S, Jian-ping J, Ning X, Zhi-mi H, Yu-yue W. Inhibitory effects of reserpine and carbonyl cyanide m3chloro3 phenylhydrazone on fluoroquinolone resistance of Acinetobacter baumannii. Chin Med J. 2005;118(4):340-43.

27. Rajamohan G, Srinivasan VB, Gebreyes WA. Novel role of Acinetobacter baumannii RND efflux transporters in mediating decreased susceptibility to biocides. J Antimicrob Chemother. 2010;65(2):228-32. 